\title{
Are DNA Repair Factors Promising Biomarkers for Personalized Therapy in Gastric Cancer?
}

Tarek Abdel-Fatah,, ${ }^{1, \star}$ Arvind Arora, ${ }^{2, \star}$ Ipek Gorguc, ${ }^{2}$ Rachel Abbotts, ${ }^{2}$ Sarah Beebeejaun, ${ }^{3}$ Sarah Storr, ${ }^{2}$ Vivek Mohan, ${ }^{2}$ Claire Hawkes, ${ }^{3}$ Irshad Soomro, ${ }^{3}$ Dileep N. Lobo, ${ }^{4}$ Simon L. Parsons, ${ }^{5}$ and Srinivasan Madhusudan ${ }^{2}$

\section{Abstract}

Chronic inflammation is a driving force for gastric carcinogenesis. Reactive oxygen species (ROS) generated during the inflammatory process generates DNA damage that is processed through the DNA repair pathways. In this study, we profiled key DNA repair proteins (single-strand-selective monofunctional uracil-DNA glycosylase 1 [SMUG1], Flap endonuclease 1 [FEN1], X-ray repair cross-complementing gene 1 [XRCC1], and Ataxia telangiectasia mutated [ATM]) involved in ROS-induced oxidative DNA damage repair in gastric cancer and correlated to clinicopathological outcomes. High expression of SMUG1, FEN1, and XRCC1 correlated to high T-stage (T3/T4) ( $p$-values: 0.001, 0.005, and 0.02, respectively). High expression of XRCC1 and FEN1 also correlated to lymph node-positive disease ( $p$-values: 0.009 and 0.02 , respectively). High expression of XRCC1, FEN1, and SMUG1 correlated with poor disease-specific survival (DSS) ( $p$-values: 0.001, 0.006, and 0.05, respectively) and poor disease-free survival (DFS) ( $p$-values: $0.001,0.001$, and 0.02 , respectively). Low expression of ATM correlated to lymph node positivity $(p=0.03)$, vascular invasion $(p=0.05)$, and perineural invasion $(p=0.005)$ and poor DFS $(p=0.001)$ and poor DSS $(p=0.003)$. In the multivariate Cox model, high XRCC1 and low ATM were independently associated with poor survival ( $p=0.008$ and 0.011 , respectively). Our observation supports the hypothesis that DNA repair factors are promising biomarkers for personalized therapy in gastric cancer. Antioxid. Redox Signal. 18, 2392-2398.

\section{Introduction}

$\mathrm{C}$ HRONIC INFLAMMATION IS a driving force for gastric carcinogenesis (2). Reactive oxygen species (ROS) generated during the inflammatory process generate DNA damaging lesions such as apurinic/apyrimidinic (AP) sites, oxidized purines, and pyrimidines (such as 8-oxo-dG), DNA single strand breaks (SSBs), oxidative-clustered DNA lesions, and DNA double-strand breaks (DSBs) in cells. If unrepaired, such DNA damaging lesions lead to accumulation of mutations and eventually cancer (6). Interestingly, in cancer cells, oncogenic transformation, enhanced metabolic activity, and increased generation of ROS also result in substantial oxidative stress compared to normal cells. Moreover tumor hypoxia and an acidic tumor microenvironment may also promote oxidative stress in cancer cells (7). Given the prevalence of ROS in the tumor microenvironment, it is perhaps not surprising that cancer cells, similar to normal cells, utilize adaptive mechanisms to maintain cellular survival. Besides the glutathione and thioredoxin systems, cells also upregulate antioxidant enzymes such as superoxide dismutases, catalases, and peroxidases to process ROS (6). However, cancer cells have a limited ROS buffering capacity, and ROS accumulation eventually leads to cellular injury and accumulation of DNA damage. Similar to normal cells, cancer cells utilize the DNA repair machinery, although less efficiently, to process damaging DNA lesions to maintain cellular survival (6).

Base excision repair (BER) is required for the accurate removal of bases that have been damage by alkylation or

\footnotetext{
${ }^{1}$ Department of Clinical Oncology, City Hospital Campus, Nottingham University Hospitals, Nottingham, United Kingdom.

${ }^{2}$ Academic Unit of Oncology, School of Molecular Medical Sciences, University of Nottingham, City Hospital Campus, Nottingham University Hospitals, Nottingham, United Kingdom.

${ }^{3}$ Department of Histopathology, Nottingham University Hospitals, Nottingham, United Kingdom.

${ }^{4}$ Division of Gastrointestinal Surgery, Nottingham Digestive Diseases Centre, NIHR Biomedical Research Unit, Nottingham University Hospitals, Nottingham, United Kingdom.

${ }^{5}$ Department of Surgery, Nottingham University Hospitals, City Hospital, Nottingham, United Kingdom.

*These two authors contributed equally to this work.
} 


\section{Innovation}

Reactive oxygen species (ROS) generated during inflammation induces DNA damage that is processed through DNA repair in cells. Whereas suboptimal DNA repair may predispose to cancer, evolving data also suggest that established tumors are under enormous oxidative stress, and dysregulation of ROS-induced DNA damage repair may in fact be essential for cancer cell survival. To test this hypothesis, the authors profiled DNA repair proteins and demonstrated that overexpression of singlestrand-selective monofunctional uracil-DNA glycosylase 1 (SMUG1), Flap endonuclease 1 (FEN1), and X-ray repair cross-complementing gene 1 (XRCC1) and downregulation of Ataxia telangiectasia mutated (ATM) correlate to adverse clinicopathological features in patients. The data suggest that ROS-induced DNA repair factors are promising biomarkers for personalized therapy in gastric cancer.

oxidation (5). BER is performed by two major subpathways: the short-patch pathway (SP-BER) and long-patch pathway (LP-BER). The subpathways differ from each other in the length of the repair patch and in the subsets of enzymes involved. However, both pathways are initiated by a damage-specific DNA glycosylase, which removes the damaged base creating an abasic site (apurinic/apyrimidinic, AP site). An AP endonuclease then cleaves the phosphodiester bond $5^{\prime}$ to the AP site, thereby generating a nick with $5^{\prime}$-sugar phosphate (dRP) and 3'-hydroxyl group. DNA polymerase $\beta$ adds the first nucleotide to the $3^{\prime}$-end of the incised AP site. Normally, the reaction continues through the short-patch repair pathway where $\operatorname{Pol} \beta$ removes the 5 '-sugar phosphate residue (by the process of $\beta$-elimination) and DNA ligase III-X-ray repair cross-complementing gene 1 (XRCC1) heterodimer (or DNA ligase I) then completes the repair. However, if the $5^{\prime}$-sugar phosphate is resistant to $\beta$-elimination (such as due to oxidation), additional DNA synthesis is then required and the repair process proceeds through the LP-BER. In this pathway, replication factor C loads proliferating cell nuclear antigen (PCNA) onto the DNA. PCNA functions as a DNA sliding clamp for Pol $\delta / \varepsilon$, which performs DNA synthesis to displace the $5^{\prime}$-sugar phosphate as part of a flap. The flap is then removed by flap endonuclease 1 (FEN1), and DNA ligase I completes long-patch repair by ligating the DNA ends (5).

ROS can also generate DNA DSBs either directly or when a replication fork encounters a SSB generated during oxidative base damage repair. Ataxia telangiectasia mutated (ATM) is critical for DNA DSB sensing and signal transduction. Evolving evidence also suggests that ATM is also a sensor of oxidative stress in cells. Moreover, ROS-induced ATM activation may also be independent of DSBs. ATM signaling under oxidative stress may alter cellular metabolism, promote cell survival, and induce expression of antioxidant enzymes to maintain redox homeostasis (3).

Given the essential role of DNA repair in oxidative stress and ROS-induced DNA damage, we hypothesized that DNA repair may be dysregulated in human gastric adenocarcinomas. Here, we have investigated the expression of
Table 1. Patients' Demographics

\begin{tabular}{lc}
\hline & Number $(\%)$ \\
\hline Total number of patient & 142 \\
Median age & 74 years \\
Sex & $105(73.9 \%)$ \\
Male & $37(26 \%)$ \\
Female & \\
T stage & $14(9.8 \%)$ \\
T1 & $48(33.8 \%)$ \\
T2 & $75(52.8 \%)$ \\
T3 & $5(3.5 \%)$ \\
T4 & \\
N stage & $33(23.2 \%)$ \\
N0 & $109(76.8 \%)$ \\
$\geq N 1$ & $140(98.5 \%)$ \\
M stage & $2(1.4 \%)$ \\
M0 & \\
M1 & $142(100 \%)$ \\
Tumor type & \\
Adenocarcinoma & $142(100 \%)$ \\
Site of tumor & \\
Gastric & $70(49 \%)$ \\
Surgery & $52(37 \%)$ \\
Total gastrectomy & $20(14 \%)$ \\
Partial gastrectomy & \\
Oesophago-gastrectomy & $54(38 \%)$ \\
Survival status & $87(62 \%)$ \\
Alive & \\
Dead & \\
\hline
\end{tabular}

single-strand-selective monofunctional uracil-DNA glycosylase 1 (SMUG1), XRCC1, FEN1, and ATM in gastric cancer patients.

Patient demographics are summarized in Table 1. A total of 142 patients underwent primary surgery for gastric adenocarcinoma. Median age was 74 years and $74 \%$ were male. Majority of tumors were T3 (53\%); 77\% of tumors were N1 or greater disease; $49 \%$ of patients underwent total gastrectomy; $62 \%$ patients were dead and $38 \%$ cases were alive at the time of analysis.

\section{Single-Strand-Selective Monofunctional Uracil-DNA Glycosylase 1}

SMUG1 glycosylase is a key enzyme for repairing 5-hydroxymethyluracil, 5 formyluracil, 5,6-dihydrouracil, alloxan, and other lesions generated during oxidative base damage (1). We investigated SMUG1 expression in gastric cancers. A total of 112 cores were suitable for analyses. About $38 / 112(34 \%)$ showed negative SMUG1 expression and 74/112 (66\%) showed positive SMUG1 expression (Fig. 1) (Table 2). SMUG1-positive tumors were more likely to be high T stage (T3 or T4) $(p=0.02)$. Patients whose tumors were SMUG1-positive had poor adverse clinical outcome. The mean disease-specific survival (DSS) in SMUG1-positive patients was 49.1 months (95\% confidence interval [CI] 38.6-59.6) compared to 60.09 months (95\% CI 47.6-72.5) in SMUG1-negative patients $(p=0.05)$ (Fig. 2A). The mean disease free survival (DFS) in SMUG1-positive patients was 47.3 months (95\% CI 36.24-59.4) compared to 62.2 months (95\% CI 50.0-74.4) in SMUG-1-negative patients $(p=0.02)$ (Fig. 2B). 


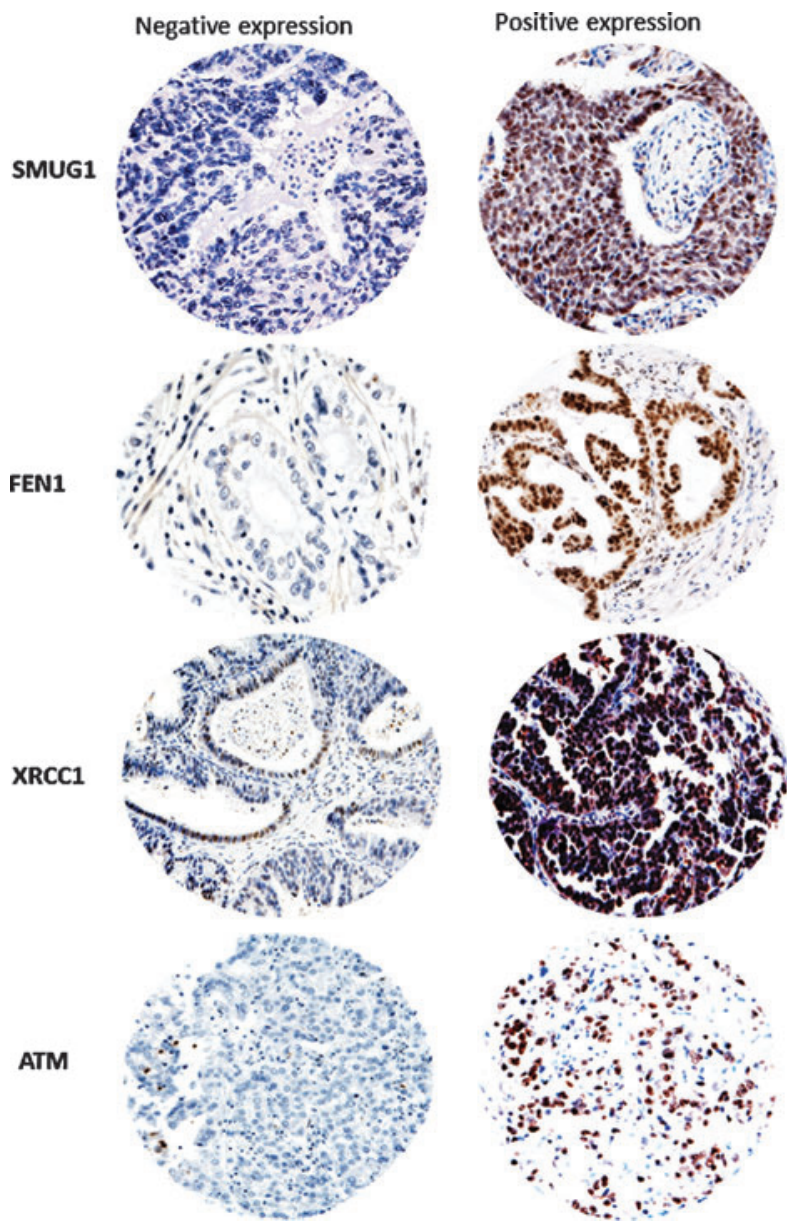

FIG. 1. Microphotographs of tumors expressing high levels of (A) SMUG1, (B) FEN1, (C) XRCC1, and (D) ATM (magnification $\times \mathbf{1 0 0}$ ). See methods for details. SMUG1, single-strand-selective monofunctional uracil-DNA glycosylase 1; FEN1, Flap endonuclease 1; XRCC1, X-ray repair crosscomplementing gene 1; ATM, Ataxia telangiectasia mutated. To see this illustration in color, the reader is referred to the web version of this article at www.liebertpub.com/ars

\section{Flap Endonuclease 1}

FEN1 structure-specific endonuclease is essential for longpatch BER involved in processing oxidative base damage in cells (9). A total of 127 cores were suitable for FEN1 analyses. About 53/127 (42\%) showed negative FEN1 expression and $74 / 127(58 \%$ ) showed positive FEN1 expression (Fig. 1) (Table 2). FEN1-positive tumors were more likely to be high $\mathrm{T}$ stage (T3 or T4) $(p=0.005)$ with lymph node positivity $(p=0.02)$. The mean DSS in FEN1-positive patients was 42.6 months (95\% CI 33.3-52.0) compared to 62.9 months (95\% CI 52.773.1) in FEN1-negative patients $(p=0.006)$ (Fig. 2D). The mean DFS in FEN1-positive patients was 39.8 months (95\% CI 29.6-49.9) compared to 71.4 months (95\% CI 60.0-82.8) in FEN1-negative patients $(p=0.001)$ (Fig. 2C).

\section{X-Ray Repair Cross-Complementing Gene 1}

XRCC1 plays a critical role in BER (5). XRCC1, a 70-kDa protein, has three functional domains: an N-terminal DNA binding domain, a centrally located BRCT I domain, and a C-terminal BRCT II domain. Although XRCC1 has no known enzymatic activity, it functions as a molecular scaffold protein and is intimately involved in the coordination of DNA repair by interacting with several components of the BER/SSBR pathway such as DNA glycosylases, apurinic/ apyrimidinic endonulcease (APE1), PARP-1, polynulceotide kinase, and ligase III involved in the repair of oxidative base damage (5). A total of 106 cores were suitable for XRCC1 analyses. About 66/106 (62\%) showed negative XRCC1 expression and 40/106 (38\%) showed positive XRCC1 expression (Fig. 1) (Table 2). XRCC1-positive tumors were more likely to be high T stage (T3 or T4) $(p=0.009)$ and were lymph node-positive $(p=0.001)$. The mean DSS in XRCC1positive patients was 30.3 months (95\% CI 21.3-39.4) compared to 63.5 months (95\% CI 52.9-74.2) in XRCC1-negative patients $(p=0.001)$ (Fig. 3A).The mean DFS in XRCC1positive patients was 29.7 months (95\% CI 20.5-38.9) compared to 64.02 months (95\% CI 52.85-75.2) in XRCC1negative patients $(p=0.001)$ (Fig. 3B).

Table 2. Clinicopathological Correlations

\begin{tabular}{|c|c|c|c|c|c|c|c|c|c|c|c|c|}
\hline \multirow[b]{2}{*}{ Variable } & \multicolumn{3}{|c|}{ SMUG 1} & \multicolumn{3}{|c|}{ FEN1 } & \multicolumn{3}{|c|}{ XRCC1 } & \multicolumn{3}{|c|}{ ATM } \\
\hline & Low & High & $\underset{\text { Value }}{\mathrm{p}-}$ & Low & High & $\begin{array}{c}\mathrm{p}- \\
\text { Value }\end{array}$ & Low & High & $\underset{\text { Value }}{\mathrm{p}-}$ & Low & High & $\underset{\text { Value }}{\mathrm{p}-}$ \\
\hline \multicolumn{13}{|l|}{ T stage } \\
\hline T1and T2 $(n=61)$ & $21(55.3)$ & $25(33.8)$ & \multirow{2}{*}{0.02} & $32(60.4)$ & $26(35.1)$ & \multirow{2}{*}{0.005} & $35(53)$ & $8(20)$ & \multirow[t]{2}{*}{0.001} & $23(39.7)$ & $20(57.1)$ & \multirow{2}{*}{0.1} \\
\hline $\mathrm{T} 3$ and $\mathrm{T} 4(n=79)$ & $17(44.7)$ & $49(66.2)$ & & $21(39.6)$ & $48(64.9)$ & & $31(47)$ & $32(80)$ & & $35(60.3)$ & $15(42.9)$ & \\
\hline \multicolumn{13}{|c|}{$\mathrm{N}$ stage } \\
\hline N0 $(n=33)$ & $11(28.9)$ & $13(17.6)$ & \multirow{2}{*}{0.16} & $18(34)$ & $12(16.2)$ & \multirow[t]{2}{*}{0.02} & $19(28.8)$ & $3(7.5)$ & \multirow[t]{2}{*}{0.009} & $10(17.2)$ & $13(37.1)$ & \multirow{2}{*}{0.03} \\
\hline N1-3 $(n=107)$ & $27(71.1)$ & $61(82.4)$ & & $35(66)$ & $62(83.8)$ & & $47(71.2)$ & $7(92.5)$ & & $48(82.8)$ & $22(62.9)$ & \\
\hline \multicolumn{13}{|c|}{ Vascular invasion } \\
\hline Absent $(n=44)$ & $12(31.6)$ & $20(27)$ & \multirow[t]{2}{*}{0.614} & $18(34)$ & $21(28.4)$ & \multirow[t]{2}{*}{0.5} & $21(31.8)$ & $9(22.5)$ & \multirow[t]{2}{*}{0.302} & $14(24.1)$ & $15(42.9)$ & \multirow{2}{*}{0.05} \\
\hline Present $(n=96)$ & $26(68.4)$ & $54(73)$ & & $35(66)$ & $53(71.6)$ & & $45(68.2)$ & $31(77.5)$ & & $44(75.9)$ & $20(57.1)$ & \\
\hline \multicolumn{13}{|c|}{ Perineural invasion } \\
\hline Absent $(n=75)$ & $21(55.3)$ & $36(48.6)$ & \multirow{2}{*}{0.5} & $30(56.6)$ & $37(50)$ & \multirow[t]{2}{*}{0.46} & $37(56.1)$ & $16(40)$ & \multirow[t]{2}{*}{0.109} & $19(32.8)$ & $22(62.9)$ & \multirow{2}{*}{0.005} \\
\hline Present $(n=65)$ & 17 (44.7) & 38 (51.4) & & $23(43.4)$ & 37 (50) & & $29(43.9)$ & $24(60)$ & & $39(67.2)$ & 13 (37.1) & \\
\hline
\end{tabular}

SMUG1, single-strand-selective monofunctional uracil-DNA glycosylase 1; FEN1, Flap endonuclease 1; XRCC1, X-ray repair crosscomplementing gene 1; ATM, Ataxia telangiectasia mutated.

Bold numbers indicate significant $p$-values. 

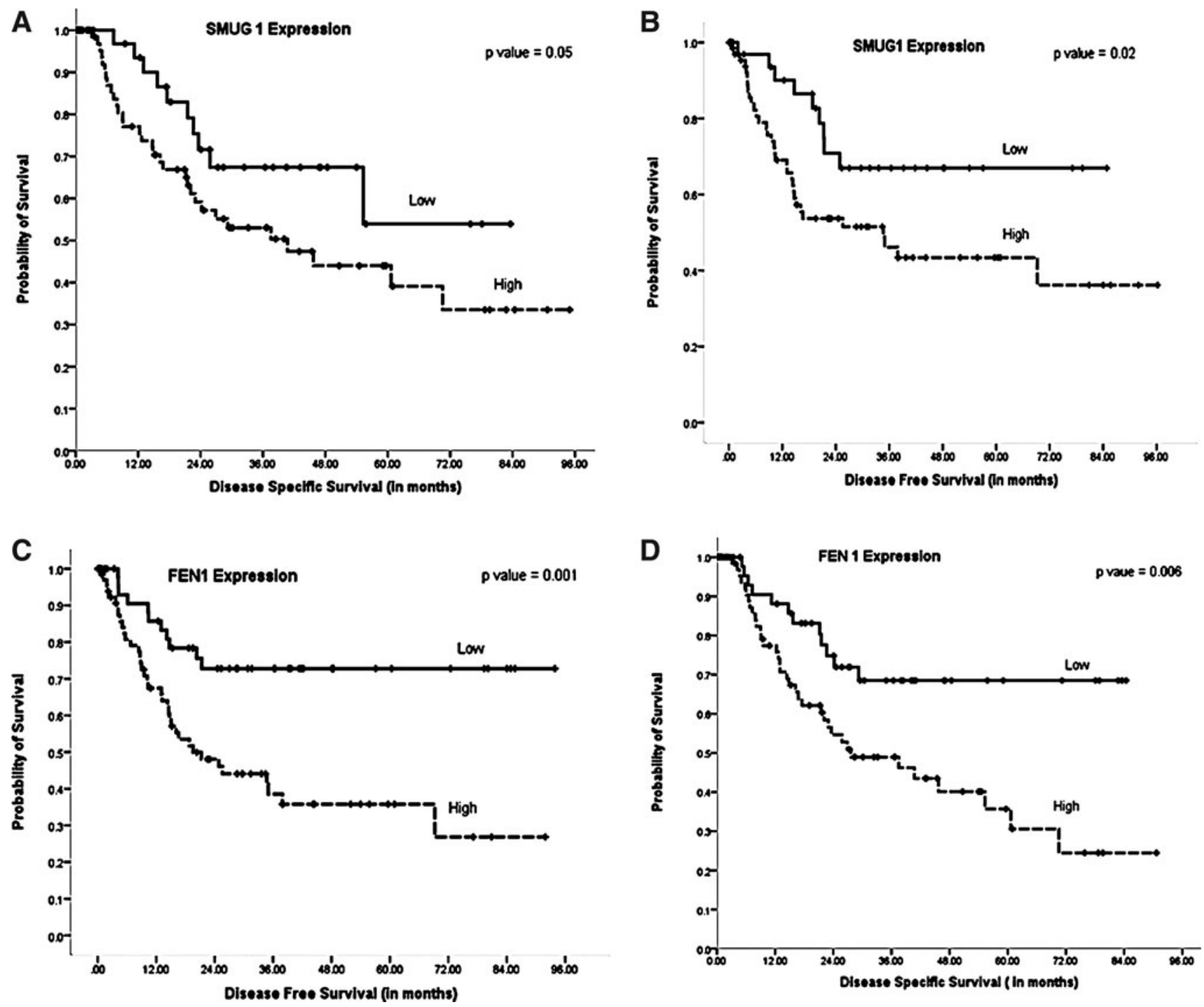

FIG. 2. Kaplan Meier curves for SMUG1 (A, DSS; B, DFS) and FEN1 (C, DSS; D, DFS). DSS, disease-specific survival (months); DFS, disease free survival (months).

\section{ATM Kinase}

ATM is critical for DNA DSB sensing and signal transduction (3). As ROS-induced DNA damage can generate DSBs, we investigated ATM expression. A total of 96 cores were suitable for ATM analyses. About 58/96 (60.5\%) showed negative ATM expression and 38/96 (39.5\%) showed positive ATM expression (Fig. 1) (Table 2). ATM-negative tumors were more likely to be lymph node-positive $(p=0.03)$ and associated with vascular invasion $(p=0.05)$ and peri-neural invasion $(p=0.005)$. The mean DSS in ATM-negative patients was 47.6 months (95\% CI 36.1-59.1) compared to 72.1 months (95\% CI 62.3-81.8) in ATM-positive patients ( $p=0.003$ ) (Fig. $3 \mathrm{C})$. The mean DFS in ATM-negative patients was 44.7 months (95\% CI 32.8-56.6) compared to 73.2 months (95\% CI 63.3-83.1) in ATM-positive patients ( $p=0.001$ ) (Fig. 3D).

\section{Multivariate Cox Regression Analysis}

Univariate analyses conducted above provide evidence that DNA repair is frequently altered in gastric cancer. To investigate whether DNA repair factors are independent biomarkers, a multivariate Cox regression analysis including other validated prognostic factors, such as T-stage, lymph node stage, and histological grade, was conducted. High XRCC1 and low ATM were powerful independent predictors for DSS ( $p=0.008$ and 0.011 , respectively) and DFS ( $p=0.032$ and 0.009 , respectively) (Table 3 ).

\section{Concluding Remarks and Future Directions}

Altered DNA repair may impact upon repair of DNA damage induced by ROS generated during inflammation in cells. Evolving evidence also suggests that dysregulated DNA repair in tumors may also affect cancer biology and select for aggressive clones with enhanced survival potential. Our data support the hypothesis that cancer cells are under considerable oxidative stress compared to normal cells and that upregulation of BER (SMUG1, FEN1, and XRCC1) is essential for the repair of oxidative base damage and survival in cancer cells. Interestingly, ATM is frequently downregulated in 
A

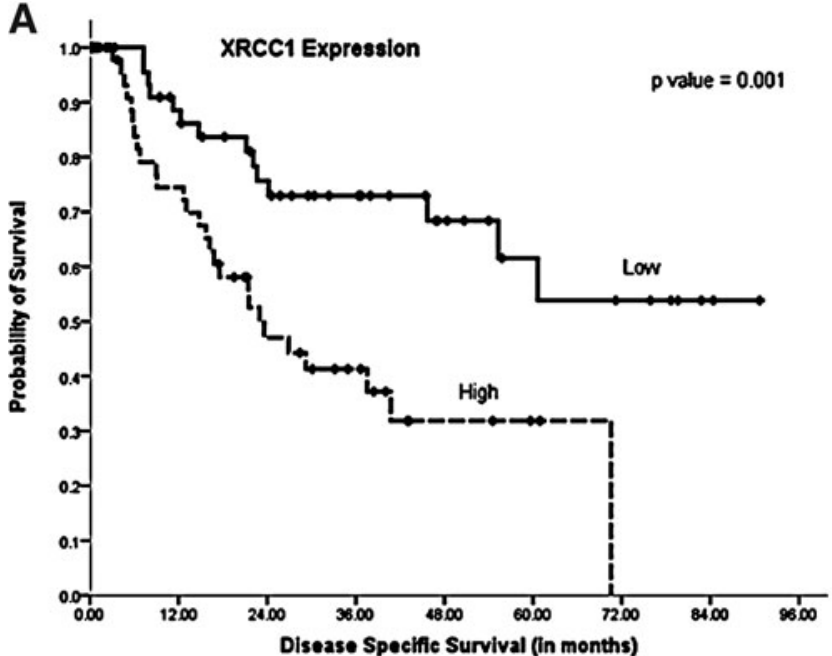

C

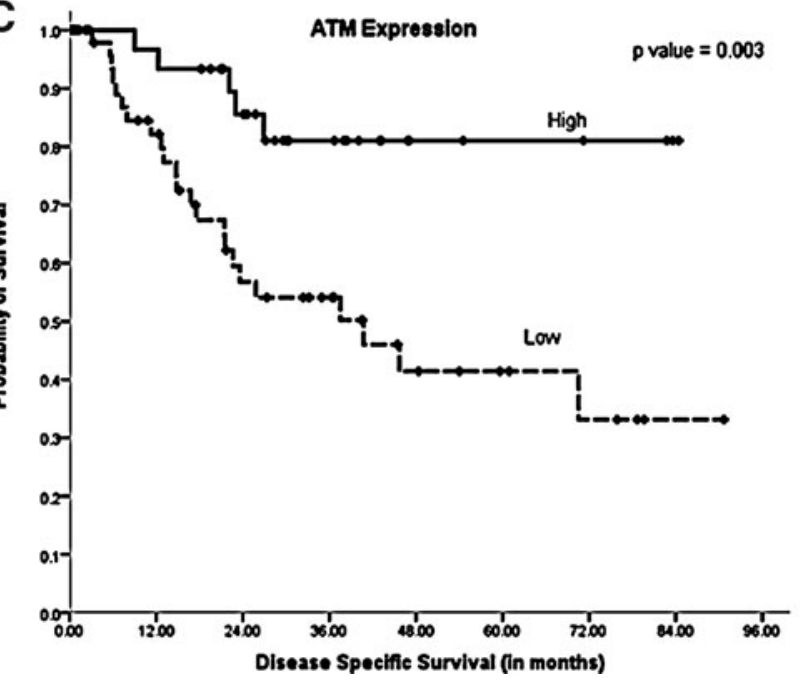

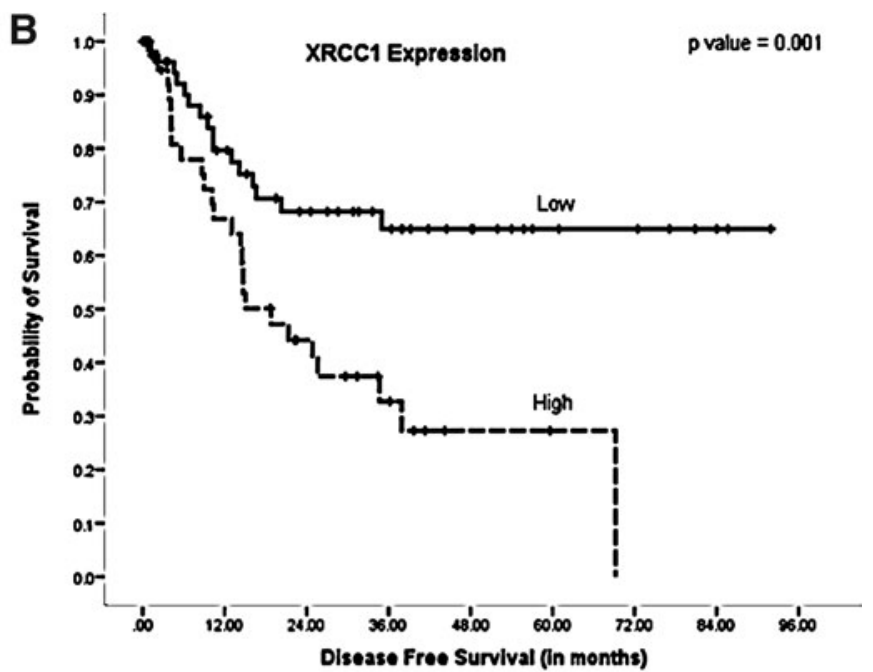

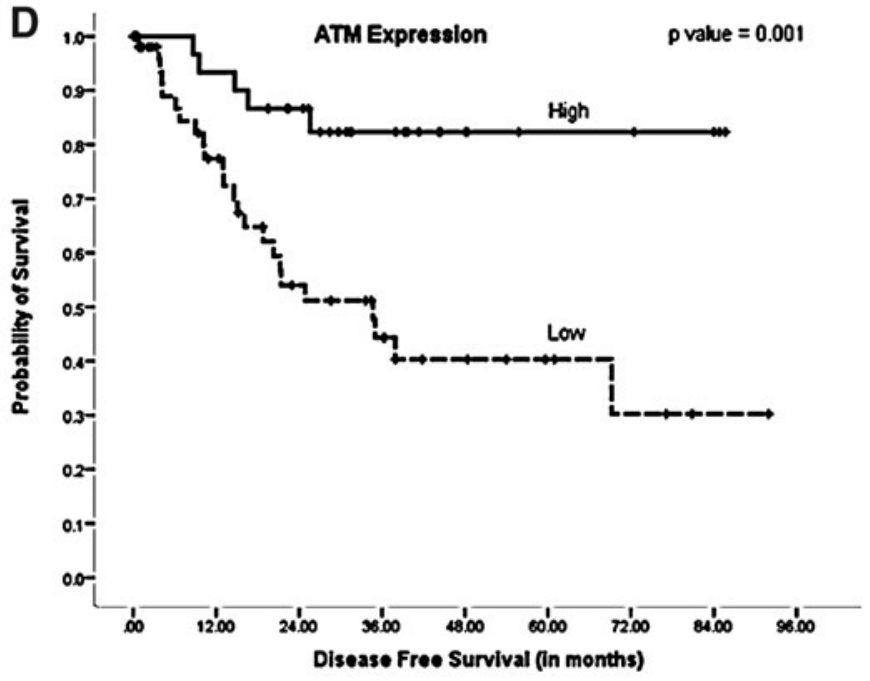

FIG. 3. Kaplan Meier curves for XRCC1 (A, DSS; B, DFS) and ATM (C, DSS; D, DFS).

Table 3. Multivariate Analysis

\begin{tabular}{lrrrr}
\hline & & & \multicolumn{2}{c}{$95 \%$ CI for $\operatorname{Exp}(B)$} \\
\cline { 5 - 5 } & & & & \\
\cline { 4 - 5 } & p-Value & Exp $(B)$ & Lower & Upper \\
\hline Disease-specific survival & & & \\
ATM & $\mathbf{0 . 0 1 1}$ & 0.227 & 0.072 & 0.711 \\
XRCC1 & $\mathbf{0 . 0 0 8}$ & 3.429 & 1.378 & 8.532 \\
SMUG1 & 0.538 & 1.410 & 0.473 & 4.208 \\
FEN1 & 0.274 & 0.535 & 0.175 & 1.639 \\
T stage & 0.100 & 2.082 & 0.870 & 4.982 \\
N stage & $\mathbf{0 . 0 0 5}$ & 2.420 & 1.312 & 4.463 \\
Grade & 0.739 & 1.107 & 0.609 & 2.011 \\
Disease free survival & & & \\
ATM & $\mathbf{0 . 0 0 9}$ & 0.240 & 0.082 & 0.701 \\
XRCC1 & $\mathbf{0 . 0 3 2}$ & 2.599 & 1.088 & 6.211 \\
SMUG1 & 0.391 & 1.594 & 0.550 & 4.626 \\
FEN1 & 0.638 & 0.773 & 0.265 & 2.258 \\
T stage & 0.147 & 1.809 & 0.811 & 4.035 \\
N stage & $\mathbf{0 . 0 1 4}$ & 2.049 & 1.159 & 3.623 \\
Grade & 0.996 & 1.001 & 0.551 & 1.819 \\
\hline
\end{tabular}

CI, confidence interval.

Bold numbers indicate significant $p$-values. gastric cancer and correlates with adverse features. Our data are also consistent with a recent study by Kang et al., who investigated ATM in 70 gastric cancers and demonstrated that low levels of ATM correlated to poor differentiation, lymph node metastasis, and poor 5-year survival (4). Taken together, the data, including ours, suggest that loss of ATM expression may be an important prognostic marker in gastric cancer. In multivariate analyses, overexpression of XRCC1 and downregulation of ATM were independently associated with poor survival. Our result is consistent with recent preclinical observations that BER and DSB repair may be differentially expressed in the tumor microenvironment. Hypoxia and acidic tumor microenvironment, besides increasing oxidative stress and inducing BER upregulation, have also been shown to suppress DSB repair, including homologous recombination (HR) (7). Our study suggests that this phenomenon may be operating gastric cancers. The ability of PARP inhibitors (that block BER and SSBR) to induce synthetic lethality in BRCAdeficient breast and ovarian cancer suggests that this approach may be feasible in other HR-deficient systems such as tumors deficient in ATM. As ATM is a key player in HR, we speculate that ATM-deficient tumors could be targeted by 
BER inhibitors. We have recently demonstrated that this approach is feasible using human APE1 inhibitors that block BER in cells (8). APE1 inhibitors were synthetically lethal in ATM-deficient cells in that study (8). The data also imply that BER inhibition such as by targeting other factors such as FEN1, SMUG1, or XRCC1 may also be an interesting future approach for personalized therapy in gastric cancer.

\section{Notes}

\section{Patients}

Investigation of the expression of SMUG1, XRCC1, FEN1, and ATM in gastro-oesophageal cancers was carried out in 142 gastric adenocarcinoma cases treated at Nottingham University Hospitals (NUH) between 2001 and 2006. Patient demographics is summarized in Table 1 . Median age was 74 years; $74 \%$ were male; $53 \%$ had T3 tumors. Patients in this cohort received primary surgery only and did not receive any adjuvant chemotherapy. Survival was calculated from the date of diagnosis until January 13, 2009, when any remaining survivors were censored. The conduct of this study was approved by the Ethics Committee of Nottingham University Hospitals.

\section{Construction of tissue microarray}

Tissue microarrays (TMAs) were constructed. In short, areaspecialized histopathologists identified and marked formalinfixed paraffin-embedded tissue blocks containing tumor tissue on hematoxylin and eosin-stained slides. The marked areas in these donor paraffin blocks were used to construct the TMA. Triplicate tissue cores with a diameter of $0.6 \mathrm{~mm}$ were taken from the marked areas and arrayed into a recipient paraffin block using a tissue puncher/arrayer (Beecher Instruments). Five-micron sections of the tissue array block were cut and placed on Fisherbrand Colorfrost/Plus microscope slides (Fisher Scientific) for immunohistochemical staining.

\section{Immunohistochemistry}

A standard streptavidin-biotin-peroxidase complex method was used. Negative controls were obtained by omitting the primary antibody in each case. The tissue slides were deparaffinized with xylene and then rehydrated through five decreasing concentrations of alcohol $(100 \%, 90 \%, 70 \%, 50 \%$, and $30 \%$ ) for $2 \mathrm{~min}$ each. Endogenous peroxidise activity was blocked by incubation in a $1 \%$ hydrogen peroxide/methanol buffer. Antigen retrieval was carried out by microwave treatment of the slides in sodium citrate buffer ( $\mathrm{pH}$ 6.0) for $10 \mathrm{~min}$. The slides were rinsed in phosphate buffer solution (PBS) and incubated with blocking serum diluted in PBS to block nonspecific staining. The following primary antibodies were used in the current study: 1/200 dilution of Goat antiSMUG1 monoclonal antibody (Acris Antibody GmbH), 1/200 dilution of anti-FEN1 rabbit polyclonal antibody (NBP167924; Novus Biologicals), 1:200 dilution of anti-XRCC1 mouse monoclonal antibody (Ab-1, clone 33-2-5; Thermoscientific), and 1/100 anti-ATM Rabbit monoclonal antibody (Clone Y170; Abcam). All primary antibody dilutions were made in PBS. After washing with PBS, sections were incubated with the secondary antibody (Vector Labs) for $30 \mathrm{~min}$ followed by the avidin-biotin complex for a further $30 \mathrm{~min}$. 3-3' Diaminobenzidine tetrahydochloride was used as a chromogen. All sections were counterstained with Gill's hematoxylin.

\section{Evaluation of immune staining}

The tumor cores were evaluated by two specialist pathologists blinded to the clinicopathological characteristics of patients, in two different settings. There was excellent intra- and interobserver agreements $(k>0.8$; Cohen's $\kappa$ and multi-rater $\kappa$ tests, respectively. Whole field inspection of the core was scored and intensities of nuclear or cytoplasmic staining were grouped as follows: $0=$ no staining, $1=$ weak staining, $2=$ moderate staining, $3=$ strong staining. The percentage of each category was estimated $(0 \%-100 \%)$. H-score (range $0-300)$ was calculated by multiplying intensity of staining and percentage staining. Low/ negative XRCC1 expression was defined by mean of H-score of $\leq 100$. Low /negative FEN1 expression was defined by mean of H-score of $\leq 100$. Low/negative SMUG1 expression was defined by mean of H-score of $\leq 100$. H-score of $>100$ was considered positive marker expression. Low/negative ATM expression was defined as $\%$ staining of $\leq 25 \%$ in tumor cores. Not all cores within the TMA were suitable for immunohistochemistry analysis due to missing cores or absence of tumor cells.

\section{Statistical analysis}

Statistical analysis of data was performed using SPSS version 20.0 for Windows (SPSS, Inc.). Univariate analysis of associations was determined using the Pearson Chi-squared $\left(\chi^{2}\right)$ test. Survival rates were calculated from the time of diagnosis until the end of the follow up period and Kaplan Meier curves were plotted. The statistical significance of differences between survival rates was determined using the log-rank test. Survival was censored if the patient was still alive. $p$-values $<0.05$ were identified as statistically significant. Multivariate analysis was performed by Cox Proportional Hazards regression model.

\section{Author Contribution}

S.M. designed and coordinated the study. T.A.F., A.A., I.G., R. A., V. M., S.B., and S.S. performed data analysis on individual markers. C.H. performed immunohistochemical optimization and staining of markers. I.S. and T.A.F conducted immunohistochemical scoring and analyses. D.N.L. and S. L. P. were involved in setting up TMAs and reviewed data. All authors were involved in writing, editing, and approval of the manuscript.

\section{References}

1. An $\mathrm{Q}$, Robins $\mathrm{P}$, Lindahl T, and Barnes DE. 5-Fluorouracil incorporated into DNA is excised by the Smug1 DNA glycosylase to reduce drug cytotoxicity. Cancer Res 67: 940-945, 2007.

2. Balkwill $\mathrm{F}$ and Mantovani A. Inflammation and cancer: back to Virchow? Lancet 357: 539-545, 2001.

3. Chen BP, Li M, and Asaithamby A. New insights into the roles of ATM and DNA-PKcs in the cellular response to oxidative stress. Cancer Lett 2011 [Epub ahead of print]: DOI: 10.1016/j.canlet.2011.12.004.

4. Kang B, Guo RF, Tan XH, Zhao M, Tang ZB, and Lu YY. Expression status of ataxia-telangiectasia-mutated gene correlated with prognosis in advanced gastric cancer. Mutat Res 638: 17-25, 2008.

5. Kim YJ and Wilson DM, 3rd. Overview of base excision repair biochemistry. Curr Mol Pharmacol 5: 3-13.

6. Pelicano H, Carney D, and Huang P. ROS stress in cancer cells and therapeutic implications. Drug Resist Updat 7: 97-110, 2004. 
7. Seo Y and Kinsella TJ. Essential role of DNA base excision repair on survival in an acidic tumor microenvironment. Cancer Res 69: 7285-7293, 2009.

8. Sultana R, McNeill DR, Abbotts R, Mohammed MZ, Zdzienicka MZ, Qutob H, Seedhouse C, Laughton CA, Fischer PM, Patel PM, Wilson DM, 3rd, and Madhusudan S. Synthetic lethal targeting of DNA double-strand break repair deficient cells by human apurinic/apyrimidinic endonuclease inhibitors. Int J Cancer 2012 [Epub ahead of print]; DOI: 10.1002/ijc.27512.

9. Zheng L, Jia J, Finger LD, Guo Z, Zer C, and Shen B. Functional regulation of FEN1 nuclease and its link to cancer. Nucleic Acids Res 39: 781-794.

Address correspondence to: Dr. Srinivasan Madhusudan Academic Unit of Oncology School of Molecular Medical Sciences

University of Nottingham

Nottingham University Hospitals Nottingham NG51PB United Kingdom

E-mail: srinivasan.madhusudan@nottingham.ac.uk
Date of first submission to ARS Central, August 9, 2012; date of acceptance, August 16, 2012.

$\quad$ Abbreviations Used
$\mathrm{ATM}=$ Ataxia telangiectasia mutated
$\mathrm{BER}=$ base excision repair
$\mathrm{DFS}=$ disease free survival
$\mathrm{DNA}=$ deoxyribonucleic acid
$\mathrm{DSBs}=$ double strand breaks
$\mathrm{DSS}=$ disease-specific survival
$\mathrm{FEN} 1=$ Flap endonuclease 1
$\mathrm{LP}-\mathrm{BER}=$ long patch base excision repair
$\mathrm{PBS}=$ phosphate buffer solution
$\mathrm{PCNA}=$ proliferating cell nuclear antigen
$\mathrm{ROS}=$ reactive oxygen species
$\mathrm{SMUG} 1=$ single-strand-selective monofunctional
$\mathrm{SP}-\mathrm{BER}=$ shoracil-DNA glycosylase 1
$\mathrm{TMAs}=$ tissue microarrays
$\mathrm{XRCC} 1=$ X-ray repair cross-complementing gene 1

